\title{
Tumorigenicity of cancer stem-like cells derived from hepatocarcinoma is regulated by microRNA-145
}

\author{
YONGSHENG JIA $^{1,2}$, HONGLIN LIU $^{1}$, QIANSHU ZHUANG ${ }^{3}$, SHIQING XU $^{1}$, \\ ZHIHUA YANG ${ }^{4}$, JIANZHONG LI ${ }^{3}$, JINNING LOU ${ }^{1}$ and WENJIAN ZHANG ${ }^{1}$ \\ ${ }^{1}$ Institute of Clinical Medical Sciences, China-Japan Friendship Hospital, Beijing; \\ ${ }^{2}$ Medical Department of Breast Oncology, Tianjin Medical University Cancer Institute and Hospital, Tianjin; \\ ${ }^{3}$ Department of Geriatrics, The First Affiliated Hospital of Soochow University, Suzhou; ${ }^{4}$ Cancer Institute \\ (Hospital), Chinese Academy of Medical Sciences, Beijing, P.R. China
}

Received December 23, 2011; Accepted February 16, 2012

DOI: $10.3892 /$ or.2012.1701

\begin{abstract}
RNAs are implicated in cancer initiation and progression by their ability to affect the expression of genes and proteins that regulate cell proliferation and death. Recent studies found that the stem cell-related genes Sox2, Oct4 and Klf4 are among the target genes regulated by microRNA-145 (miR-145), suggesting that miR-145 possibly plays a role in the maintenance of cancer stem cells. Therefore, it is important to address the involvement of miR-145 in the key roles of cancer stem cells in cancer initiation, progression and reoccurrence. We compared miR-145 expression in the cancer stem-like cells (T3A-A3) derived from hepatocarcinoma, in the hepatocarcinoma cell line BEL-7402 and in the normal liver sinusoidal endothelial cell line (LSEC). As demonstrated by a TaqMan microRNA real-time assay, T3A-A3 cells express lower miR-145 levels compared to the other cell lines. To address the role of miR-145 in cancer stem cells, miR-145 was restored in T3A-A3 cells. This resulted in senescence-like $\mathrm{G}_{1}$ arrest in cell cycling, and significantly inhibited clonogenic cell expansion in vitro and xenograft tumor growth in vivo. Moreover, miR-145 restoration diminished tumorsphere growth of T3A-A 3 cells in vitro and T3A-A 3 cells tumor formation in nude mice in vivo. Additionally, the increase in miR-145 levels paralleled the decrease in Oct 4 levels. The effect of miR-145 on tumor suppression in T3A-A3 cells was partly reversed by overexpression of Oct 4 both in vitro and in vivo. Collectively, our data indicate that miR-145 plays an important role in cancer stem cell tumorigenicity, potentially via modulation of the downstream target, Oct4.
\end{abstract}

Correspondence to: Dr Wenjian Zhang, Institute of Clinical Medical Sciences, China-Japan Friendship Hospital, Beijing 100029, P.R. China

E-mail: zwj-72@163.com

Key words: microRNA, cancer stem cell, tumorigenicity, Oct4, hepatocarcinoma

\section{Introduction}

The cancer stem cell (CSC) concept suggests that CSCs are at the germinal centers of tumor evolution; similar to normal adult stem cells, CSCs possess self-renewal capacity and differentiation potential. Over the past few years, compelling evidence has emerged in support of the hierarchic cancer model for many solid tumors, including hepatocellular cancer (1). Despite the current progress in understanding the contribution of CSCs to the generation of tumor heterogeneity, the molecular complexity and exact regulation of CSCs is poorly understood. microRNAs represent a class of small non-coding RNAs that are key regulators of many cellular events, including the balance between proliferation and differentiation during tumorigenicity (2). Studies using the Dicer gene knockout mouse model demonstrate that miRNAs may be critical regulators of embryonic stem cell organogenesis $(3,4)$. Moreover, accumulated data suggest that miRNA dysregulation occurs frequently in a variety of carcinomas, including those of the lung, colon, stomach, pancreas, and liver (5). Sachdeva and Mo (6) have shown that miR-145 is underexpressed in tumor tissues and is capable of inhibiting tumor cell growth and invasion by targeting several genes such as c-Myc and mucin 1 . This unique feature of miR-145-mediated gene silencing may suggest that miR-145 is a potential cancer biomarker that could serve as a novel target for cancer therapy. Xu et al (7) revealed a direct link between the core reprogramming factors and miR-145, and uncovered a double-negative feedback loop involving Oct4, Sox2, Klf4 and miR-145. The dual effects of miR-145 in both carcinogenesis and differentiation of normal stem cells strongly suggest that they may be involved in the maintenance and survival of cancer stem cells. For this reason, we undertook the current study to investigate the potential of miR-145 in regulating the tumorigenicity of cancer stem-like cells.

\section{Materials and methods}

Cell lines. Cancer stem-like cells (T3A-A3) were isolated from human liver cancer tissue and purified by single cell 
colony screening and tumorigenicity screening as previously described (8).

The hepatocarcinoma cell line BEL-7402, the immortalized liver sinusoidal endothelial cell line LSEC, and HEK293T cells were maintained in our laboratory. BEL-7402 cells were cultured in RPMI-1640. All other cell lines were routinely cultured in DMEM supplemented with $10 \%$ heat-inactivated fetal bovine serum (Gibco, USA), $100 \mathrm{U} / \mathrm{ml}$ penicillin (Sigma, USA), $100 \mu \mathrm{g} / \mathrm{ml}$ streptomycin (Sigma) and maintained in the presence of $5 \% \mathrm{CO}_{2}$ at $37^{\circ} \mathrm{C}$.

Flow cytometry. Cells were incubated in phosphate-buffered saline (PBS) containing 2\% fetal bovine serum (FBS) and $0.1 \%$ sodium azide with phycoerythrin (PE)-conjugated CD133 (Miltenyi Biotec, Auburn, CA, USA). Isotype-matched mouse immunoglobulins served as controls. Samples were analyzed using a Beckman-Coulter flow cytometer and the CellQuest software.

Total RNA extraction and RT-PCR. Total RNA was extracted using TRIZol (Invitrogen, Shanghai, China) according to the manufacturer's protocol. For miRNA analysis, the expression level of miR-145 was quantified by a TaqMan microRNA realtime assay that was performed by using an Applied Biosystems 7500 Sequence Detection System (Applied Biosystems). The expression of miRNAs were defined from the threshold cycle $(\mathrm{Ct})$, and relative expression levels were calculated after normalization to the expression of U6 small nuclear RNA (9). For mRNA analysis, $1 \mu \mathrm{g}$ of total RNA was used for reverse transcription by using Omniscript reverse transcriptase (Qiagen, Shanghai, China). The cDNA was amplified using the primers: AGCCCTCATTTCACCAGGCC and TGGGACTC CTCCGGGTTTTG (Oct4, 456 bp); AACAAGATGAGATT GGCATGG and AGTGGGGTGGCTTTTAGGAT ( $\beta$-actin, $251 \mathrm{bp}$ ). $\beta$-actin mRNA levels were used as an internal normalization control. The PCR products were visualized on a $1.5 \%$ agarose gel stained with ethidium bromide.

Lentivirus production and transduction. In HEK293T cells, the vector pLVTHM-scramble or pLVTHM-miR-145 (kindly provided by the Professor K.S. Kosik, University of California) were co-transfected with the envelope plasmid pMD2.G and the packing vector psPAX2 by using calcium phosphate precipitation. Virus-containing medium was collected $48 \mathrm{~h}$ after transfection and concentrated by ultracentrifugation at $28,000 \mathrm{rpm}$ for $2 \mathrm{~h}$. Consequently, concentrated viruses were reconstituted in phosphate-buffered saline (PBS). Lentiviruses containing the vector pLVTHMscramble (Lenti-scr) or pLVTHM-miR-145 (Lenti-miR-145) were produced (7). Full-length human Oct4 cDNA (kindly provided by Professor Hongkui Deng, Peking University) was amplified with the following primers: forward, GATCGGATCCATGGCGGGACACCTGGCT and reverse, TCGCAGTCGACTCAGTTTGAATGCATGGG. PCR products were ligated, digested with $B a m H I$ and $S a l \mathrm{I}$, and cloned into the site of pWPTS. The pWPTS-Oct4 lentiviruses were produced as described above.

Cell cycle analysis. T3A-A3 cells were infected with Lenti-miR-145 or Lenti-scr, trypsinized $72 \mathrm{~h}$ later, washed with PBS, and fixed in $70 \%$ ethanol on ice overnight. After centrifugation, cells were stained with $50 \mu \mathrm{g} / \mathrm{ml}$ propidium iodide (Sigma) and $0.1 \mu \mathrm{g} / \mathrm{ml}$ RNase A (Sigma), and analyzed by flow cytometry by using FACStar Plus ${ }^{\mathrm{TM}}$. Each histogram was constructed using data from at least 5,000 events. Data were analyzed to calculate the percentage of cell population in each phase.

Tumorsphere culture. T3A-A3 cells were suspended in serumfree DMEM (Invitrogen, Carlsbad, CA, USA), supplemented with B27 (1:50, Invitrogen), $20 \mathrm{ng} / \mathrm{ml}$ hEGF (Invitrogen), $20 \mathrm{ng} / \mathrm{ml}$ basic fibroblast growth factor (bFGF; R\&D Systems), $40 \mathrm{U} / \mathrm{ml}$ heparin (Sigma), 2 mM glutamine (Sigma), $100 \mathrm{U} / \mathrm{ml}$ penicillin, $100 \mu \mathrm{g} / \mathrm{ml}$ streptomycin, $5 \mu \mathrm{g} / \mathrm{ml}$ insulin (Sigma), and $0.5 \mu \mathrm{g} / \mathrm{ml}$ hydrocortisone (Sigma), and plated in 24-well ultra-low attachment plates (Corning, USA) at 2,000 cells per well. After 15-20 days, the plates were analyzed for tumorsphere formation, and quantified using an inverted microscope (IX71; Olympus, Japan).

Clonogenic formation assay. Cells $\left(1 \times 10^{3}\right)$ were plated in a 6 -well plate. After 10 days of culture, the surviving colonies ( $>50$ cells per colony) were counted with crystal violet staining. Independent experiments were performed in triplicate.

MTT assay. T3A-A3 cells were infected with Lenti-miR-145 or Lenti-scr. Oct4 expression vector was introduced into the Lenti-miR-145 culture by pWPTS-Oct4 infection. T3A-A3 cells expressing Lenti-scr, Lenti-miR-145 or Lenti-miR-145 + pWPTS-Oct4 were plated in 96-well plates (1,000 cells/well). Three wells were selected randomly on days 1,3 , and 5 for MTT (3-(4,5-dimethythiazolyl)-2,5-diphenyl-2H-tetrazolium bromide) assay. In brief, MTT $(5 \mathrm{mg} / \mathrm{ml})$ was added to each well $\left(50 \mu \mathrm{l} /\right.$ well) and incubated for $4 \mathrm{~h}$ at $37^{\circ} \mathrm{C}$, then waterinsoluble formazan dye in the cells was solubilized with dimethyl sulfoxide (DMSO) $(150 \mu \mathrm{l} /$ well), and the optical density was quantified by an ELISA reader (Multiskan MK3, Thermo Scientific, USA) at $492 \mathrm{~nm}$.

Western blot analysis. To determine protein expression levels, cells were harvested, lysed in a RIPA lysis buffer [50 mM Tris-HCl (pH 8.0), $150 \mathrm{mM} \mathrm{NaCl,} \mathrm{0.1 \%} \mathrm{SDS,} \mathrm{1 \%} \mathrm{NP-40,}$ $0.25 \%$ sodium deoxycholate, and $1 \mathrm{mM}$ EDTA] with freshly added protease inhibitor cocktail (Roche, USA) for $30 \mathrm{~min}$ on ice, and subsequently centrifuged at $13,000 \mathrm{rpm}$ for $10 \mathrm{~min}$. The total protein concentration of whole-cell extracts was measured using Bradford reagent (Bio-Rad Laboratories, Hercules, CA, USA), and proteins were resolved by SDS-PAGE (Bio-Rad Laboratories). After electrophoresis, the proteins were electrotransferred to PVDF membranes (Millipore, USA), blocked with 5\% skimmed milk, and probed with the following antibodies diluted in PBS/BSA: Oct4 (Santa Cruz Biotechnology, Santa Cruz, CA, USA) followed by horseradish peroxidase (HRP)-conjugated secondary antibody (Sigma). Binding was detected with enhanced chemiluminescence (Millipore).

Senescence-associated $\beta$-galactosidase staining. Senescenceassociated $\beta$-galactosidase staining was performed using the Senescence $\beta$-Galactosidase Staining kit (Beyotime 
Table I. In vivo tumor development experiments of T3A-A3 cells infected with Lenti-miR-145 or Lenti-scr in nude mice.

\begin{tabular}{|c|c|c|c|}
\hline Cell type ${ }^{a}$ & Cell number injected & Tumor incidence ${ }^{b}$ & Latency (days) ${ }^{c}$ \\
\hline \multirow[t]{3}{*}{ Lenti-scr } & $2 \times 10^{4}$ & $4 / 6$ & 63 \\
\hline & $2 \times 10^{5}$ & $6 / 6$ & 42 \\
\hline & $2 \times 10^{6}$ & $6 / 6$ & 15 \\
\hline \multirow[t]{3}{*}{ Lenti-miR-145 } & $2 \times 10^{4}$ & $0 / 6$ & - \\
\hline & $2 \times 10^{5}$ & $2 / 6$ & 42 \\
\hline & $2 \times 10^{6}$ & $5 / 6$ & 21 \\
\hline
\end{tabular}

${ }^{a}$ T3A-A3 cells infected with Lenti-miR-145 or Lenti-scr were injected subcutaneously in nude mice; nude mice injected with tumor cells but showing no signs of tumor burden were generally sacrificed 5 months after tumor cell inoculation. ${ }^{\text {b }}$ The number of tumors detected/number of injections. ${ }^{\mathrm{c}}$ Approximate number of days from tumor cell injection to appearance of a tumor.

Biotechnology, Haimen, China) following the manufacturer's instructions. Senescent cells were identified under a light microscope.

Fluorescence microscopy. Cells were plated on coverslips for at least $24 \mathrm{~h}$ and fixed using $4 \%$ paraformaldehyde in PBS for 15 min at room temperature. After washing with PBS, cells were permeabilized for $5 \mathrm{~min}$ on ice with $0.2 \%$ Triton X-100 in PBS with 3\% BSA (PBS/BSA). The cells were subsequently washed with PBS/BSA and incubated for $1 \mathrm{~h}$ with a primary antibody, anti-p16 (Santa Cruz Biotechnology), diluted in PBS/BSA. After washing in PBS/BSA, cells were stained with Texas Red-conjugated secondary antibodies (Invitrogen) for $30 \mathrm{~min}$ at room temperature in a humidified chamber. Finally, the cells were washed in PBS, and viewed under a IX71 fluorescence microscope.

In vivo tumor growth experiments. Five to six-week-old female athymic BALB/c nude mice were purchased from the Institute of Laboratory Animal Science, Chinese Academy of Medical Sciences. T3A-A3 cells infected with relevant lentiviruses were collected and inoculated subcutaneously into nude mice ( $n=6$ for each group) on the right flank $\left(2 \times 10^{6}\right.$ cells $/$ site $)$. Tumor sizes were measured using calipers. Tumor volumes were calculated using the following formula: (length $\mathrm{x}$ width $\left.{ }^{2}\right) / 2$. On the day indicated, all tumors were collected, and their weights were measured. All animal research was approved by our institutional Animal Ethics Committee.

In vivo tumorigenicity experiments. Various numbers of cells (Table I) were injected subcutaneously into nude mice ( $n=6$ for each group). Mice were sacrificed between 2 and 5 months post-injection, at which time the tumors were harvested for further examination. Animals injected with tumor cells but showing no signs of tumor burden were generally sacrificed 5 months after tumor cell inoculation; they were opened up at the injection sites to confirm a lack of tumor development.

Immunohistochemistry. Tumors were excised, fixed in $4 \%$ paraformaldehyde, and processed for paraffin embedding. Tumor tissue sections were immunostained using mouse anti-
Oct4 (Santa Cruz Biotechnology) or mouse anti-PCNA (Santa Cruz Biotechnology), in addition to biotinylated secondary antibodies and horseradish peroxidase-conjugated antibiotin by using an ABC-peroxidase kit (Vector Laboratories, Burlingame, CA), according to the manufacturer's protocol. The tumors were viewed under a light microscopy (Olympus IX71).

Statistical analysis. The results are expressed as mean \pm SD. Two-tailed t-tests were employed to analyze the in vitro and in vivo data using the Prism 5.0 software (GraphPad, San Diego, CA). Statistical significance was set at $\mathrm{P}<0.05$, while $\mathrm{P}<0.01$ indicated a highly significant difference.

\section{Results}

Downregulation of miR-145 in T3A-A3 cells correlates with increases in tumor growth. T3A-A 3 cells were originated from a primary human liver cancer, and were established by single cell colony screening, and tumorigenicity screening. T3A-A3 cells possessed a high tumorigenic potential and expressed classical stem cell markers. Here we show that T3A-A3 cells express high levels of a liver cancer stem cell maker (CD133) $(10,11)$ compared with the hepatocarcinoma cell line BEL-7402, and the liver sinusoidal endothelial cells, LSEC (Fig. 1A). Then we compared miR-145 expression in the three cell lines by using the TaqMan microRNA real-time assay. As shown in Fig. 1B, LSEC cells expressed high levels of miR-145, while T3A-A3 cells expressed very low levels. As shown in Fig. 1C, LSEC and BEL-7402 cells formed fewer tumorspheres than T3A-A3 cells. The tumorigenicity of the three cell lines was assessed in a nude mouse xenograft model. Thirty days after subcutaneous inoculation into female nude mice, the tumor volumes of T3A-A 3 cells were significantly greater than those of BEL-7402 cells; no tumor formation was detected in LSEC cells (Fig. 1D). Since miR-145 deficiency is correlated with an increase in tumor growth, this prompted us to investigate whether miR-145 functions as a tumor suppressor in T3A-A3 cells.

miR-145 restoration in T3A-A3 cells results in $G_{1}$ arrest and growth inhibition. To address the effect of miR-145 
A

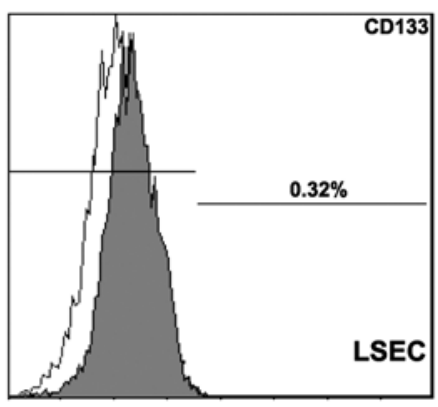

B

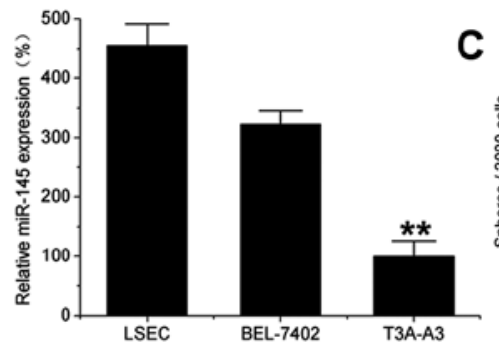

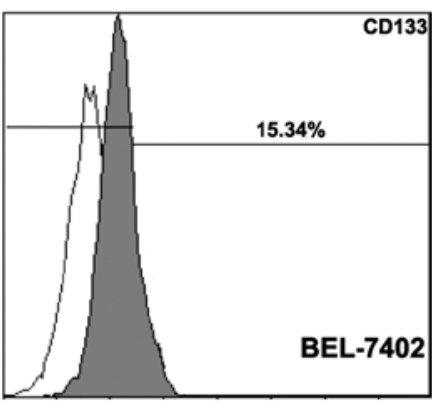

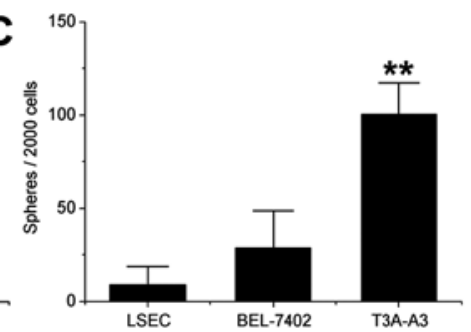

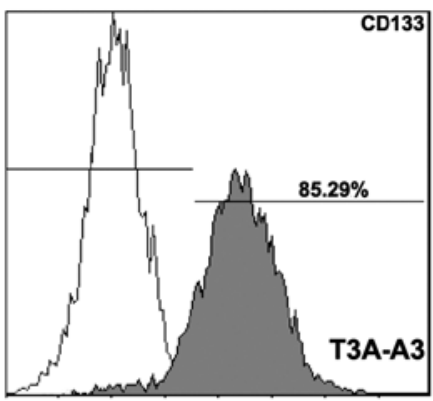

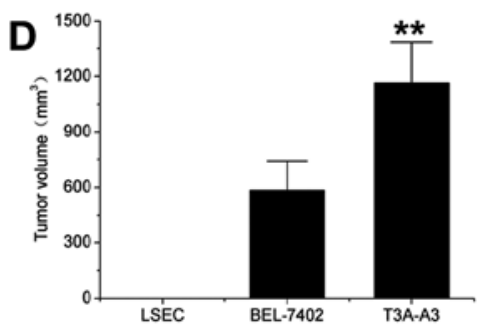

Figure 1. Downregulation of miR-145 in T3A-A3 cells correlates with increases in tumor growth. (A) CD133 FACS analysis of the LSEC, BEL-7402, and T3A-A3 cells. (B) Comparison of the expression levels of miR-145 in LSEC, BEL-7402, and T3A-A3 cells by TaqMan real-time PCR. U6 snRNA was used as an endogenous control. ${ }^{* *} \mathrm{P}<0.01$ for T3A-A3 vs. LSEC and T3A-A3 vs. BEL-7402, $\mathrm{n}=3$. (C) LSEC, BEL-7402, and T3A-A3 cells were plated for tumorsphere formation. After 15-20 days, tumorspheres were observed under a microscope and quantified. ${ }^{* *} \mathrm{P}<0.01$ vs. BEL-7402, $\mathrm{n}=3$. (D) Cells were collected and subcutaneously inoculated $\left(6 \times 10^{6}\right.$ cells $\left./ 0.2 \mathrm{ml}\right)$ into nude mice on 1 side of the flank $(\mathrm{n}=6)$. Tumor volume was measured thirty days after inoculation. ${ }^{* *} \mathrm{P}<0.01$ for T3A-A3 vs. BEL-7402 cells.

A

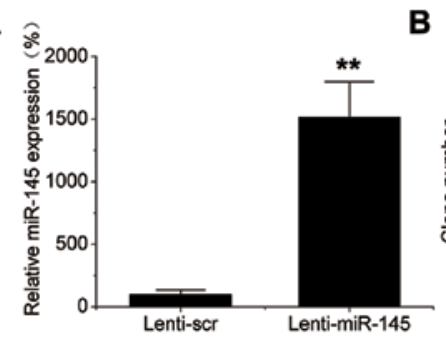

D

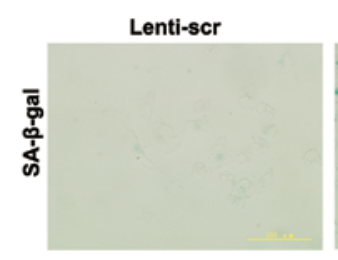

$\mathbf{F}$

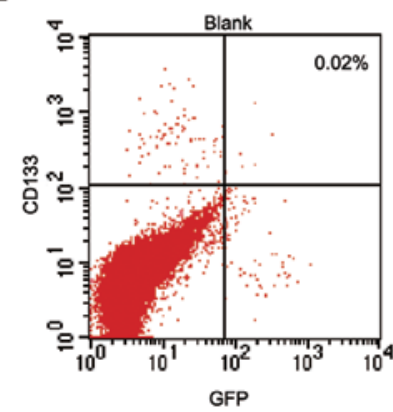

B

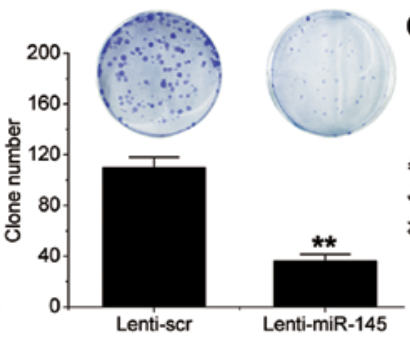

$\mathbf{E}$

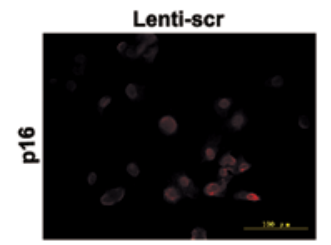

Lenti-miR-145
C

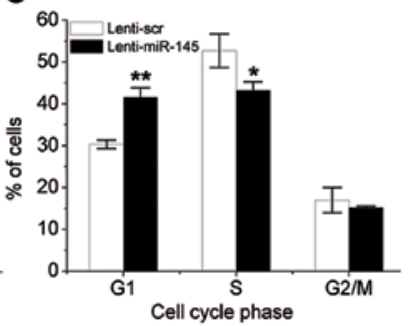

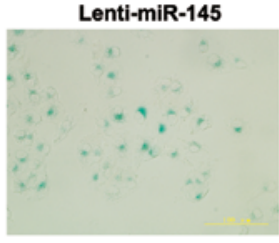
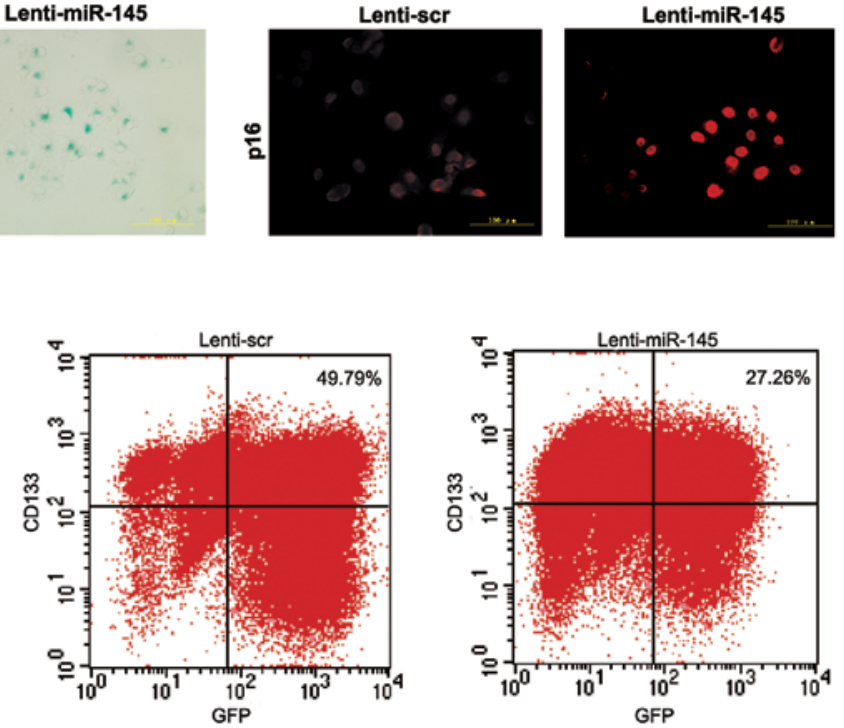

Figure 2. miR-145 restoration in T3A-A3 cells induces $\mathrm{G}_{1}$ arrest and growth inhibition. (A) miR-145 expression in T3A-A3 cells infected with Lenti-miR-145 or Lenti-scr for $72 \mathrm{~h}$ detected by TaqMan real-time PCR. ${ }^{* *} \mathrm{P}<0.01$ for Lenti-miR-145 vs. Lenti-scr, $\mathrm{n}=3$ (Lenti-scr, T3A-A3 cells infected with negative control lentivirus vector; Lenti-miR-145, T3A-A3 cells infected with miR-145 lentivirus). (B) Representative inhibition of clonogenic formation by Lenti-miR-145 in T3A-A3 cells. ${ }^{* *} \mathrm{P}<0.01$ vs. Lenti-scr, $\mathrm{n}=3$. (C) Cell cycle analysis $72 \mathrm{~h}$ post infection. ${ }^{*} \mathrm{P}<0.05,{ }^{* *} \mathrm{P}<0.01$ vs. Lenti-scr, $\mathrm{n}=3$. (D) Cells were stained for $\mathrm{SA}-\beta$-gal 3 weeks post-infection. Representative photomicrographs are shown; senescent cells exhibit cytoplasmic blue staining. (E) p16 expression was detected by immunofluorescence 3 weeks post-infection. (F) CD133 FACS analysis of T3A-A3 cells after miR-145 retoration. 

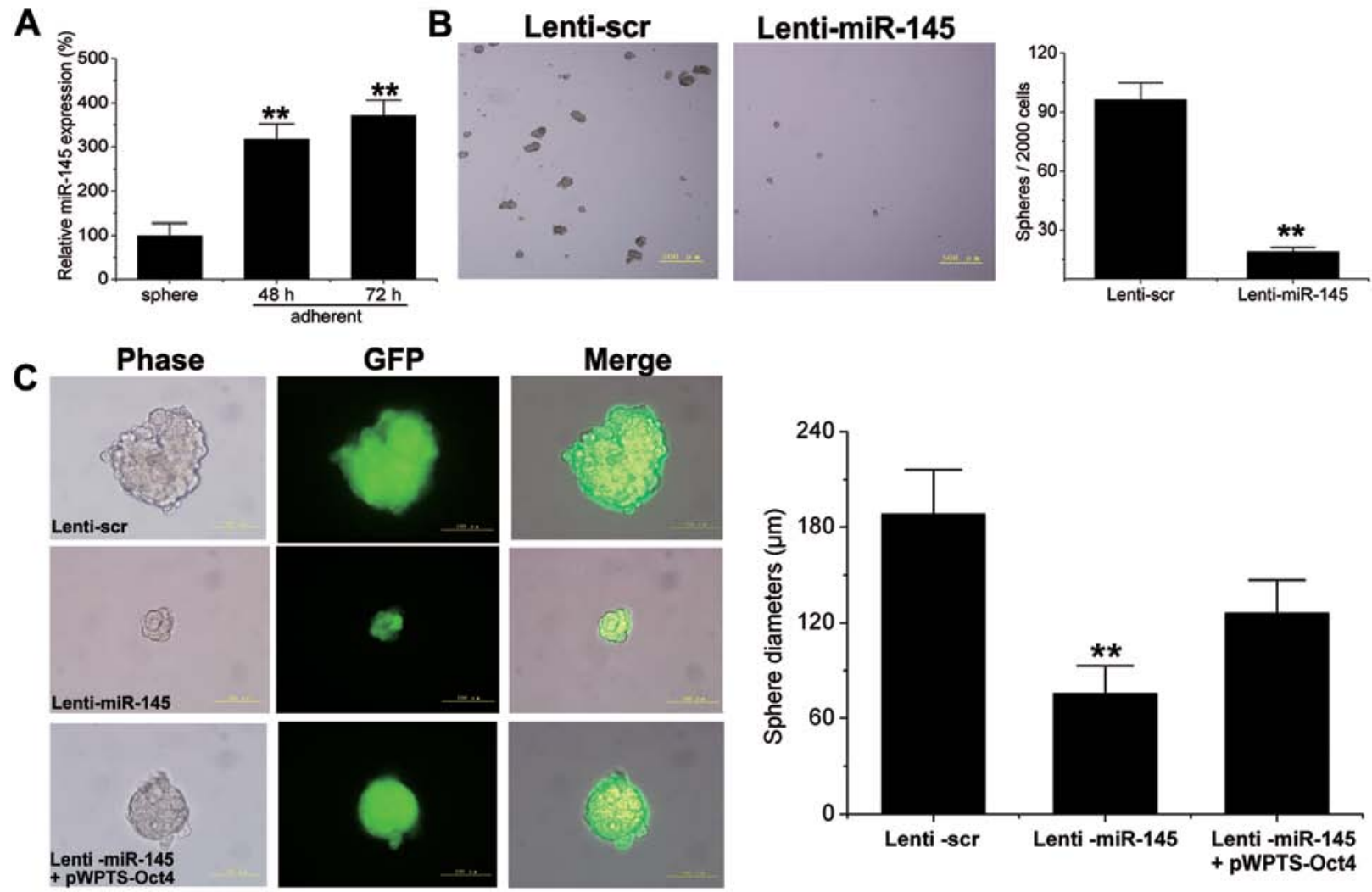

Figure 3. miR-145 restoration inhibits T3A-A3 tumorsphere formation. (A) Comparison of miR-145 expression in T3A-A3 cells cultured under tumorspheric and adherent conditions. ${ }^{* *} \mathrm{P}<0.01$ vs. sphere, $\mathrm{n}=3$. (B) T3A-A3 cells infected with Lenti-miR-145 or Lenti-scr were plated for tumorsphere formation. After 15-20 days, tumorspheres were observed under a microscope and quantified. ${ }^{* *} \mathrm{P}<0.01$ vs. Lenti-scr, $\mathrm{n}=3$. (C) Tumorspheres generated from single-cell cultures of T3A-A3 infected with Lenti-scr, Lenti-miR-145 or Lenti-miR-145 + pWPTS-Oct4 were imaged on day 30 of suspension culture. The sphere diameters are expressed as mean $\pm \mathrm{SD}$. ${ }^{* *} \mathrm{P}<0.01$ vs. Lenti-scr, $\mathrm{n}=10$.

on the function of T3A-A3 cells, we infected T3A-A3 cells with Lenti-miR-145. After 72 h miR-145 expression levels were determined by TaqMan microRNA real-time assay. As expected, miR-145 expression levels were significantly elevated by Lenti-miR-145 infection (Fig. 2A). After confirming that the infected miR-145 lentiviruses were functional, we carried out a clonogenic assay to examine the effects of miR-145 restoration on cell growth. As shown in Fig. 2B, miR-145 restoration significantly inhibited clonogenic cell growth, inhibiting colony formation by $67 \%$ compared to Lenti-scr ( $36.5 \pm 4.9$ vs. $110 \pm 8.1$ colonies/well, respectively). To analyze the mechanism by which miR-145 restoration inhibits cell proliferation, cell cycle distributions were detected by FACS. The results showed that cells infected with Lenti-miR-145 had significantly larger $G_{1}$ populations and smaller $S$ populations compared to the Lenti-scr cells; the difference regarding the percentage of cells in the $G_{2}$ phase between the treated and control groups was not significant (Fig. 2C). When the clonal cultures were passaged for 3 weeks, Lenti-scr-infected cells proliferated extensively and exhibited a typical 'cobblestonelike' epithelial morphology, whereas Lenti-miR-145-infected cells became large, flat, and senescent, as evidenced by positive SA- $\beta$-gal staining (Fig. 2D) and p16 immunofluorescence (Fig. 2E). As a maker of liver cancer stem cells, CD133 positive hepatocellular carcinoma cells possess high capacity for selfrenewal. As shown in Fig. 2F the expression levels of CD133 in T3A-A3 cells were significantly lowered after miR-145 restoration, providing a clue to the relationship between selfrenewal and miR-145 in T3A-A3 cells.

Reduced miR-145 levels are important to maintain tumorspheres. Tumorsphere culturing, wherein cells grow in suspension under non-adherent culture conditions to become tumorspheres, is widely used to assess the self-renewal potential of stem cells and cancer stem cells (12). We found that tumorspheric T3A-A3 cells had reduced miR-145 expression as compared with adherent T3A-A3 cells (Fig. 3A). Therefore, the effects of miR-145 restoration on tumorsphere formation were evaluated. As shown in Fig. 3B, T3A-A3 cells infected with Lenti-miR-145 formed 5.1-fold fewer tumorspheres than T3A-A3 cells infected with Lenti-scr; the tumorspheres were significantly smaller than those of the controls. This result was confirmed by a separate tumorsphere study in a 96-well-based single-cell tumorsphere culture, in which the tumorspheres were found to be from single cells, and not from cell aggregates (Fig. 3C). Our data provide evidence that Lenti-miR-145 inhibits tumorsphere formation and growth in cancer stem-like cells, implying that miR-145 may play a role in the self-renewal of cancer stem-like cells.

miR-145 restoration inhibits T3A-A3 tumor development in nude mice. To determine whether miR-145 restoration inhibits T3A-A3 tumor formation in vivo, we carried out tumor development experiments by using T3A-A 3 cells infected with 
A

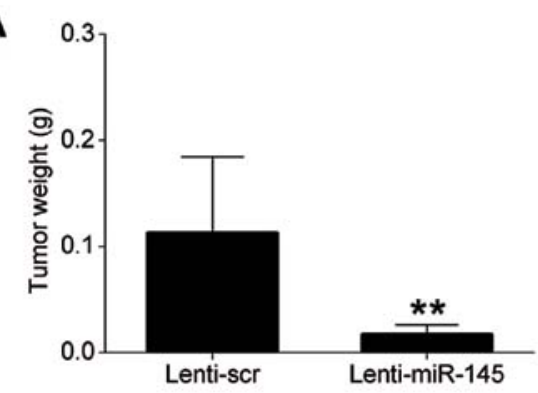

C

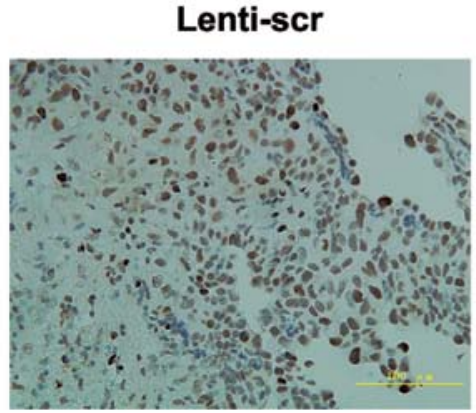

B

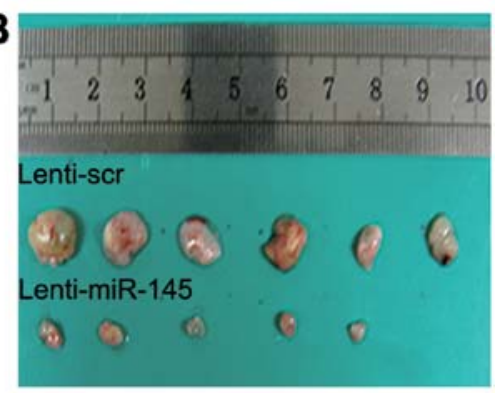

\section{Lenti-miR-145}

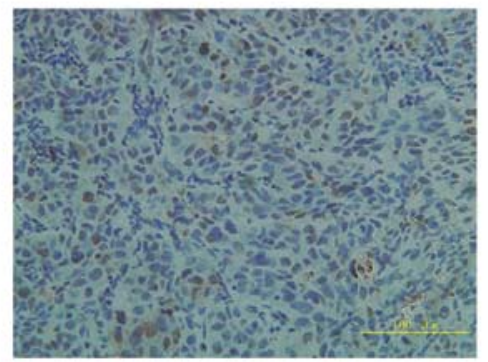

Figure 4. miR-145 restoration inhibits T3A-A3 tumor growth. (A) The effect of miR-145 restoration on xenograft tumor growth. Cells were collected and inoculated subcutaneously $\left(2 \times 10^{6}\right.$ cells $\left./ 0.2 \mathrm{ml}\right)$ into nude mice on 1 side of the flank. On Day 33, all tumors were collected to measure the tumor weights. ${ }^{* *} \mathrm{P}<0.01$ vs. Lenti-miR-145, $\mathrm{n}=6$. (B) Tumors formed by T3A-A3 infected with Lenti-145 or Lenti-scr 33 days after inoculation. (C) PCNA staining indicates that Lenti-scr tumors have a higher proliferative index than Lenti-145 tumors.

A

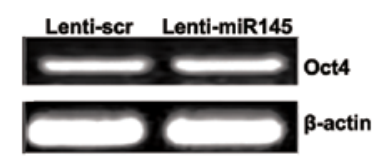

C

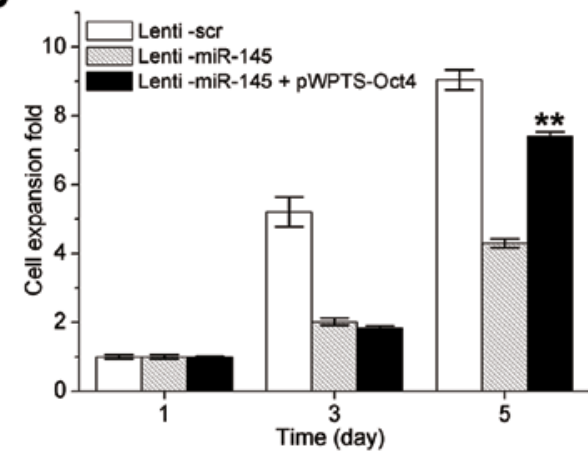

B

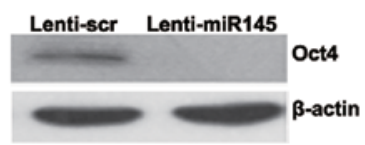

D

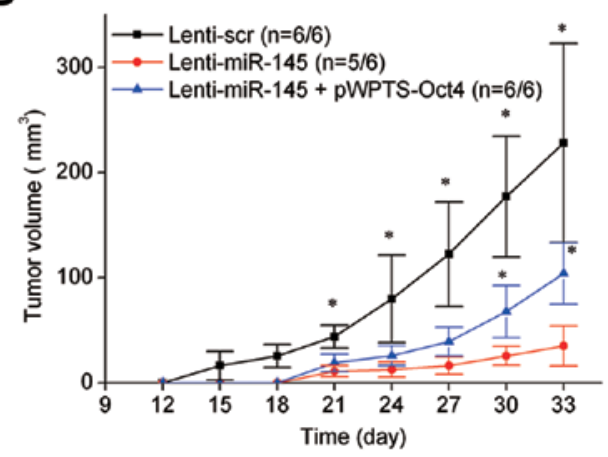

Figure 5. The tumor suppression effects of Lenti-miR-145 were partly abolished by pWPTS-Oct4. (A) T3A-A3 cells were infected with Lenti-miR-145 or Lenti-scr for $72 \mathrm{~h}$; Oct 4 expression was detected by RT-PCR and normalized to that of $\beta$-actin. (B) T3A-A3 cells were infected with Lenti-miR-145 or Lenti-scr for $72 \mathrm{~h}$; Oct 4 expression was detected by western blotting and normalized to that of $\beta$-actin. (C) The cell expansion inhibitory effects of Lenti-miR-145 were partly abolished by pWPTS-Oct4. ${ }^{* *} \mathrm{P}<0.01$ vs. Lenti-miR-145, $\mathrm{n}=3$. (D) The tumor growth inhibitory effects of Lenti-miR-145 were partly abolished by pWPTS-Oct4. Tumor sizes were measured using calipers every three days. Tumor volumes were calculated using the following formula: (length $\mathrm{x}$ width $\left.{ }^{2}\right) / 2$. $\mathrm{n}=$ detections/number of injections. ${ }^{* *} \mathrm{P}<0.01$ vs. Lenti-miR-145, $\mathrm{n}=6$.

Lenti-miR-145 or Lenti-scr. Infected cells were inoculated subcutaneously in nude mice (Table I). A significant difference in tumor incidence was observed between Lenti-miR-145 and Lenti-scr T3A-A3 cells. As few as $2 \times 10^{4}$ Lenti-scr T3A-A3 cells were sufficient for consistent tumor development in nude mice, whereas at least 10 times as many Lenti-miR-145 T3A-A3 cells were necessary to consistently generate tumors in the same model. Moreover, the Lenti-miR-145 expressing tumors grew more slowly than the control, and the average tumor weight was significantly smaller than that of the control (Fig. 4A). In keeping with their slow growth, a lower proportion of Lenti-miR-145-expressing tumor cells than Lenti-scr-expressing tumor cells stained for proliferating cell associated antigen (PCNA) (Fig. 4C). 
The tumor suppression effects of Lenti-miR-145 are partly abolished by pWPTS-Oct 4 . We examined the expression levels of Oct 4 , which has been identified as one of targets of miR-145 in human embryonic stem cells. As shown in Fig. 5A and B, the levels of Oct4 protein but not mRNA were inversely correlated with miR-145 expression. Then Oct4 expression vector was introduced into the Lenti-miR-145 culture by pWPTS-Oct 4 infection, and the cell proliferation in vitro and tumor growth in vivo were evaluated. Interestingly, the inhibition effect of Lenti-miR-145 on T3A-A 3 cells were partly abrogated by pWPTS-Oct 4 both in vitro (Figs. $3 \mathrm{C}$ and $5 \mathrm{C}$ ) and in vivo (Fig. 5D). These results suggest that miR-145 is involved in the tumorigenesis of cancer stem-like cells, potentially via the direct modulation of the downstream target Oct4.

\section{Discussion}

Therapies that target CSCs may have unique properties compared to those targeting the bulk of a tumor. From this perspective, effective cancer treatment requires targeting CSCs. However, we must determine the signals that regulate CSCs survival and function, and identify whether there is an effective way to subvert them. miRNAs are important regulators of gene expression. In principle, they can contribute to tumorigenesis by modulating the oncogenic and tumor suppressor pathways (13). Here, we found that miR-145 is underexpressed in the cancer stem-like T3A-A3 cells compared to the hepatocarcinoma BEL-7402 cells and the liver sinusoidal endothelial LSEC cells. Moreover, miR-145 deficiency was correlated with an increase in CD133 expression and tumor development. However, the mechanisms by which miR-145 influences cancer development are yet unclear. Thus, a gain-of-function analysis by lentivirus infection was used to detect the possible roles and related mechanisms by which miR-145 regulates the tumorigenicity of the cancer stem-like cells.

It is widely accepted that cancer cells require unlimited replicative potential to generate macroscopic tumors. This capability sharply contrasts with the behavior of cells in most normal cell lineages in the body, which are able to pass only through a limited number of successive cell growth and division cycles (14). This limitation is associated with a distinct barrier to proliferation: senescence. Cellular senescence, a growth-arresting program that limits the lifespan of mammalian cells and prevents unlimited cell proliferation, is attracting considerable interest because of its relation to tumor suppression. In our study, miR-145 restoration in the cancer stem-like cells significantly inhibited clonogenic cell growth and induced $\mathrm{G}_{1}$ arrest and senescence. These results are consistent with the findings of Bonifacio and Jarstfer (15), according to which miR-145 was upregulated by approximately 3.5 -fold during senescence. Thus, we postulate that miR-145 restoration-induced cellular senescence may contribute to its tumor suppression capability in cancer stem-like cells.

Oct4 belongs to a family of transcription factors containing the POU DNA-binding domain. Oct4 expression can be detected in the embryonic stem cells as well as in the adult stem cells, such as the bone marrow-derived mesenchymal stem cells (16). Oct4 plays a pivotal role in maintaining the pluripotency and self-renewal of the embryonic stem cells. Ectopic expression of this factor in adult mice has been found to cause the formation of dysplastic lesions in the skin and intestines (17). Chen et al (18) showed that Oct4 expression maintains cancer stem-like properties in lung cancer-derived CD133-positive cells. Oct4 has been identified as a direct target of miR-145 in human embryonic stem cells (7); we confirmed these findings in T3A-A3 cells, wherein the level of Oct 4 protein but not mRNA was inversely correlated with miR-145 expression. In fact, we showed that miR-145 restoration significantly inhibited tumorsphere growth in vitro as well as tumor formation in vivo. Underlying these hallmarks is the tumor suppression effect of miR-145, which was partly abolished by Oct 4 both in vitro and in vivo. Thus, our data support the view that miR-145 plays an important role in the tumorigenesis of cancer stem-like cells, potentially via the direct modulation of the downstream target, Oct4. These findings suggest that miR-145 may hold significant promise as a novel molecular therapy for hepatocarcinoma.

\section{Acknowledgements}

This study was supported by the National Basic Research Program of China (2009CB521804). We thank Professor K.S. Kosik of the Neuroscience Research Institute at the University of California for kindly providing the pLVTHM-scramble and pLVTHM-miR-145 vectors. We thank Professor Hongkui Deng of the College of Life Sciences at Peking University for kindly providing the full-length human Oct 4 cDNA.

\section{References}

1. Marquardt JU, Factor VM and Thorgeirsson SS: Epigenetic regulation of cancer stem cells in liver cancer: current concepts and clinical implications. J Hepatol 53: 568-577, 2010.

2. Calin GA and Croce CM: MicroRNA signatures in human cancers. Nat Rev Cancer 6: 857-866, 2008.

3. Bibikova M, Laurent LC, Ren B, Loring JF and Fan JB: Unraveling epigenetic regulation in embryonic stem cells. Cell Stem Cell 2: 123-134, 2008.

4. Laurent LC, Chen J, Ulitsky I, Mueller FJ, Lu C, Shamir R, Fan JB and Loring JF: Comprehensive microRNA profiling reveals a unique human embryonic stem cell signature dominated by a single seed sequence. Stem Cells 26: 1506-1516, 2008.

5. Ladeiro Y, Couchy G, Balabaud C, Bioulac-Sage P, Pelletier L, Rebouissou S and Zucman-Rossi J: MicroRNA profiling in hepatocellular tumors is associated with clinical features and oncogene/tumor suppressor gene mutations. Hepatology 47: 1955-1963, 2008.

6. Sachdeva M and Mo YY: miR-145-mediated suppression of cell growth, invasion and metastasis. Am J Transl Res 2: 170-180, 2010.

7. Xu N, Papagiannakopoulos T, Pan G, Thomson JA and Kosik KS: MicroRNA-145 regulates OCT4, SOX2, and KLF4 and represses pluripotency in human embryonic stem cells. Cell 137: 647-658, 2009.

8. Jia Y, Zhang W, Liu H, Peng L, Yang Z and Lou J: Inhibition of glutathione synthesis reverses Krüppel-like factor 4-mediated cisplatin resistance. Cancer Chemother Pharmacol 69: 377-385, 2012.

9. Wang S, Bian C, Yang Z, Bo Y, Li J,Zeng L, Zhou H and Zhao RC: miR-145 inhibits breast cancer cell growth through RTKN. Int J Oncol 34: 1461-1466, 2009.

10. Ma S, Chan KW, Hu L, Lee TK, Wo JY, Ng IO, Zheng BJ and Guan XY: Identification and characterization of tumorigenic liver cancer stem/progenitor cells. Gastroenterology 132: 2542-2556, 2007.

11. Yin S, Li J, Hu C, Chen X, Yao M, Yan M, Jiang G, Ge C, Xie H, Wan D, Yang S, Zheng S and Gu J: CD133 positive hepatocellular carcinoma cells possess high capacity for tumorigenicity. Int J Cancer 120: 1444-1450, 2007.

12. Yu F, Yao H, Zhu P, Zhang X, Pan Q, Gong C, Huang Y, Hu X, Su F, Lieberman J and Song E: let-7 regulates self renewal and tumorigenicity of breast cancer cells. Cell 131: 1109-1123, 2007. 
13. Ventura A and Jacks T: microRNAs and cancer: short RNAs go a long way. Cell 136: 586-591, 2009.

14. Hanahan D and Weinberg RA: Hallmarks of cancer: the next generation. Cell 144: 646-674, 2011.

15. Bonifacio LN and Jarstfer MB: MiRNA profile associated with replicative senescence, extended cell culture, and ectopic telomerase expression in human foreskin fibroblasts. PLoS One 5: e12519, 2010.

16. Butteroni C, De Felici M, Scholer HR and Pesce M: Phage display screening reveals an association between germline-specific transcription factor Oct-4 and multiple cellular proteins. J Mol Biol 304: 529-540, 2000.
17. Hochedlinger K, Yamada Y, Beard C and Jaenisch R: Ectopic expression of Oct-4 blocks progenitor-cell differentiation and causes dysplasia in epithelial tissues. Cell 121: 465-477, 2005.

18. Chen YC, Hsu HS, Chen YW, Tsai TH, How CK, Wang CY, Hung SC, Chang YL, Tsai ML, Lee YY, Ku HH and Chiou SH: Oct-4 expression maintained cancer stem-like properties in lung cancer-derived CD133-positive cells. PLoS One 3: e2637, 2008. 